\title{
Evaluation Du Traitement Chirurgical Des Fractures De Type Burst Non Deficitaire
}

\author{
Essossinam Kpelao \\ Service de neurochirurgie du CHU de Grenoble (Michallon) \\ Grenoble cedex \\ Service de neurochirurgie, CHU Sylvanus Olympio, Lomé - Togo \\ Mikael Meyer
}

Service de neurochirurgie du CHU de Grenoble (Michallon), Grenoble cedex

Vivien Mendes Martins

Service de neurochirurgie du Centre hospitalier Régional d'Annecy.

Metz-Tessy

\section{Jean-Paul Chirossel}

Service de neurochirurgie du CHU de Grenoble (Michallon), Grenoble cedex Tchin Darre

Service de neurochirurgie, CHU Sylvanus Olympio, Lomé - Togo

\section{Emmanuel Gay}

Service de neurochirurgie du CHU de Grenoble (Michallon), Grenoble cedex

doi: 10.19044/esj.2016.v12n9p100 느:http://dx.doi.org/10.19044/esj.2016.v12n9p100

\begin{abstract}
Introduction: The difficulty in treating of burst fracture is marked by secondary kyphosis. We expose our results in this challenge.

Material and Methods: We retrospectively evaluated on preoperative, postoperative, and follow up scanners of 40 patients operated between 2007 and 2012 in the neurosurgery department of the CHU Grenoble these parameters: vertebral kyphosis (CV), regional kyphosis (CR), traumatic regional angulation (ART) and the inter-pedicular distance. The follow-up should be at least a year.

Results: The mean age of patients was 39.2 years. Average postoperative distraction was $5.8 \mathrm{~mm}$, and angular reduction was $8.5^{\circ}$ on the $\mathrm{CV}$ and $9.8^{\circ}$ on ART. At follow-up, the loss of correction was respectively $3.2^{\circ}$ and $6.1^{\circ}$ on CV and ART. The secondary compaction was $2.5 \mathrm{~mm}$. Secondary regional kyphosis was higher in short instrumentation $\left(7^{\circ}\right.$ against $\left.5.2^{\circ}\right)$ as well as for patients with a BMI $\geq 25$.
\end{abstract}


Conclusion: the evolution after treatment of burst fracture is to secondary kyphosis, despite a low functional impact. The vertebro / kyphoplasty is a complementary alternative.

Keywords: Evaluation- Surgical treatment - Burst fracture

\section{Résumé}

Introduction : Les difficultés thérapeutiques des fractures de type « burst » résident dans la restauration et le maintien de la statique vertébrale à long terme. Nous exposons nos résultats dans ce challenge.

Matériel et méthodes : Nous avons évalués rétrospectivement sur les scanners préopératoire, postopératoire et au recul de 40 patients opérés entre 2007 et 2012 dans le service de neurochirurgie du CHU Grenoble les cyphoses vertébrale $(\mathrm{CV})$ et régionale $(\mathrm{CR})$; l'angulation régionale traumatique (ART) et la distance inter-pédiculaire. Le recul devrait être au moins d'une année.

Résultats : L'âge moyen des patients était de 39,2 ans. En post-opératoire la distraction était en moyenne de $5,8 \mathrm{~mm}$ avec un gain respectif de $8,5^{\circ}$ sur la $\mathrm{CV}$ et de $9,8^{\circ}$ sur l'ART. Au recul, on notait une perte de correction de $3,2^{\circ}$ pour la $\mathrm{CV}$ et $6,1^{\circ}$ pour l'ART avec un tassement secondaire de $2,5 \mathrm{~mm}$. La cyphose régionale secondaire était plus importante en cas de montage court $\left(7^{\circ}\right.$ contre $\left.5,2^{\circ}\right)$ de même que pour les patients avec un IMC $\geq 25$.

Conclusion : Le traitement des fractures de type burst expose à une cyphose secondaire, même s'il existe un faible retentissement clinique. La vertébro/kyphoplastie constitue une option thérapeutique complémentaire.

Mots clés : Èvaluation- Traitement chirurgical - fracture type burst.

\section{Introduction}

Les fractures de type «burst» sont définies comme des fractures touchant les colonnes antérieure et moyenne du rachis par un mécanisme de compression prédominante, et sont caractérisées par un éclatement avec rétropulsion du mur postérieur dans le canal rachidien (Denis, 1983 ; Wilcox, 2003). Cette fracture correspond au type A 3 de la classification de Magerl (Magerl, 1994). Elles représentent 10- 20\% des fractures et prédominent au niveau de la charnière thoraco-lombaire (Denis, 1983 ; Been, 1999 ; Muller, 1999). Leur difficulté thérapeutique réside dans la restauration et le maintien de la statique vertébrale à long terme; car ces fractures possèdent un caractère évolutif non négligeable après le traitement. Si les abords antérieurs semblent donner les meilleurs résultats anatomiques; le peu de retentissement clinique des angulations secondaires associé à la lourdeur technique de ces abords font opter pour d'autres alternatives avec des 
résultats similaires (Muller, 1999 ; Esses 1990 ; Chritodoulou, 2005). Dans cette optique, nous avons voulu évaluer les résultats de notre pratique dans la prise en charge de ces fractures sans trouble neurologique.

\section{Matériel et méthodes}

Il s'agissait d'une étude rétrospective portant sur tous les patients opérés entre 2007 et 2012 dans le service de neurochirurgie du CHU de Grenoble pour une fracture de type "burst» de la charnière dorsolombaire non déficitaire. Au moins 2 des 3 scanners utiles à l'étude (préopératoire, post-opératoire, au recul) devaient être nécessairement réalisés dans le service de radiologie de l'hôpital. Les principaux paramètres étudiés étaient : La cyphose vertébrale (CV), la cyphose régionale (CR), l'angulation régionale traumatique (ART), la distance inter pédiculaire (DIP) des vertèbres adjacentes à celle fracturée (figure 1), les signes cliniques au recul (gibbosité et douleur). Le recul devrait être au moins d'une année.

\section{Résultats}

Nous avons colligé 40 patients, 28 hommes (70\%) et 12 femmes (30\%). L'âge moyen était de 39,2 ans avec des extrêmes de 13 et 55 ans. Les étiologies étaient dominées par les accidents de sport (45\%). Les vertèbres L1 (45\%) et T12 (25\%) étaient les plus concernées. Tous les patients ont été opérés en première intention par voie postérieure avec laminectomie. L'ostéosynthèse par un montage long était mise dans $62,5 \%$. Aucun déficit post-opératoire n'a été noté. Dix-sept patients avaient un scanner pré et postopératoire permettant d'évaluer la qualité de la réduction. La distraction était en moyenne de $5,8 \mathrm{~mm}$ avec un gain de $8,5^{\circ}$ sur la $\mathrm{CV}$ et de $9,8^{\circ}$ sur l'ART. Au cours du suivi, 2 patients ont présenté une gibbosité clinique, et 8 une douleur rachidienne (modérée chez 6 et invalidante chez 2 patients). Trente patients (30) avaient un scanner post-opératoire et au recul, permettant d'analyser l'évolution des paramètres post-opératoires. On notait une perte de correction de $3,2^{\circ}$ pour la $\mathrm{CV}$ et de $6,1^{\circ}$ pour $1^{\prime} \mathrm{ART}$ avec un tassement secondaire de $2,5 \mathrm{~mm}$. La perte de correction était supérieure en cas de montage court que long ( $7^{\circ}$ contre $5,2^{\circ}$ sur l'ART) (Tableau I). Les patients avec un IMC supérieur à 25 avaient une cyphose légèrement supérieure à celle des patients avec un poids normal (Tableau II). Deux patients ont bénéficié d'un complément chirurgical par abord antérieur, un d'une vertébroplastie et 3 ont été réopérés pour défaillance du matériel d'ostéosynthèse.

\section{Discussion}

Notre échantillon a été réduit par l'impossibilité d'obtenir pour chaque patient, les scanners exigés car, environ 400 patients étaient opérés 
pendant cette période pour ce type de fracture dans le service. En effet, la majorité des scanners initiaux et au recul n'étaient pas réalisés dans notre centre, empêchant ainsi les comparaisons.

Notre attitude thérapeutique, en première intention dans les burst est une voie postérieure avec greffe postéro-latérale. Cette technique nous a permis d'obtenir en post-opératoire, une $\mathrm{CV}$ et un ART moyen respectif de $5,4^{\circ}$ et $8,3^{\circ}$ similaires aux données de la littérature (Muller, 1999 ; Argenson, 1996). La meilleure réduction est celle dont l'ART est proche de $0^{\circ}$, seul garant de son maintien à long terme. Mais cet objectif est difficile à atteindre dans les burst par voie postérieure à cause de la comminution fracturaire, et par l'impossibilité de reconstitution de la colonne antérieure (Muller, 1999 ; Wang, 2008). De plus, un recul du mur postérieur de plus de $50^{\circ}$ est en général associé à une rupture du ligament vertébral postérieur et à une rotation du fragment qui compromettent sa réduction par ligamentotaxis (mise en tension tu LVCP par distraction et par extension) (Willen, 1990).

$\mathrm{Au}$ recul, la perte de réduction était de $3,2^{\circ}$ pour la $\mathrm{CV}$ et de $6,1^{\circ}$ pour l'ART. Ces valeurs se trouvent dans la moyenne d'autres travaux (Argenson, 1996 ; Lindsey, 1991). Cette perte reste supérieure à celles survenant dans les montages antérieur ou combiné (Defno, 1998 ; Haas, 1991). La cyphose secondaire est due à une insuffisance de la colonne antérieure avec augmentation des contraintes sur le montage expliquant l'incidence élevée des fractures du matériel d'ostéosynthèse (Been, 1999; Muller, 1999 ; Hitchon, 2006). Nous en avons eu deux (02) dans notre série. Une étude multicentrique (Argenson, 1996) a révélé pour les voies postérieures que ni la greffe postérolatérale, ni le montage long, ni le port de corset post-opératoire n'avait d'influence significative sur le maintien de la réduction. Par contre, les thérapeutiques complémentaires telles les vertébroplasties et kyphoplasties existent pour minimiser cette cyphose secondaire (Christodoulou, 2005 ; Defino, 1998 ; Lazennec, 2006). Il faut noter cependant que la corrélation entre la cyphose secondaire et son impact sur les scores fonctionnels restent controversés (Willen, 1990; Lazannec, 2006).

Par la mesure de la distance inter-pédiculaire (DIP), nous avons voulu savoir s'il pouvait exister un tassement vertical pur; par un glissement secondaire des vis sur les tiges. L'idée finale était de savoir si on avait une perte de la distraction per-opératoire. Mais au vue de la faible variation de la DIP $(2,5 \mathrm{~mm})$, nous pensons qu'il s'agit plutôt de la conséquence de la cyphose secondaire sur l'ensemble du montage qu'un tassement vertical.

Le choix du montage est fonction du degré de comminution de la vertèbre, du niveau lésionnel et des équipes. Le montage court a l'avantage d'immobiliser le moins de segments vertébraux, de raccourcir le délai 
opératoire et de minimiser les pertes sanguines, mais il est associé dans 9$54 \%$ des cas à une défaillance de matériel et à une cyphose secondaire en absence de reconstruction du corps vertébral (Alvine, 2004). Les montages longs permettent de minimiser cette perte de correction sans l'empêcher (Argenson, 1996). Dans notre série, la perte de réduction était plus importante pour les montages courts de l'ordre de $2^{\circ}$ sur l'ART.

Nous avons voulu également relever l'influence du poids sur la perte de réduction à long terme. Dans notre série, elle était légèrement supérieure dans le groupe des patients avec un IMC supérieur à 25. L'effet précis du poids sur la colonne vertébrale n'est pas encore totalement élucidé. Cependant, il est clair que l'obésité entraine une augmentation de la charge sur la colonne vertébrale avec accélération des lésions arthrosiques et des déformations (Chen, 2011; Hangai, 2008). Très peu d'études se sont intéressées aux influences du poids sur les montages d'ostéosynthèse en traumatologie. Les résultats obtenus après la correction de la scoliose ne semblent pas montrer de différence significative des angulations entre obèses et non obèses (Chen, 2011 ; Upasani 2008). Mais une étude multicentrique randomisée serait nécessaire pour trancher sur cette question.

\section{Conclusion}

La voie postérieure seule, peut ne pas suffire dans certains cas dans le traitement des fractures de type burst à cause des cyphoses secondaires, même s'il existe un faible retentissement sur le plan clinique. La vertébro et kyphoplastie nous paraissent un complément idéal surtout chez les sujets jeunes. Une étude prospective, multicentrique devrait être menée pour préciser l'influence réelle du poids sur les montages d'ostéosynthèse.

\section{Déclarations}

Conflits d'intérêts : Les auteurs ne déclarent aucun conflit d'intérêts

Contributions des auteurs: tous les auteurs ont contribué à la réalisation de l'étude. Tous les auteurs ont lu et approuvé la version finale du manuscrit.

\section{References:}

Alvine GF, Swain JM, Asher MA et al. (2004). Treatment of thoracolumbar burst fractures with variable screw placement or Isola instrumentation and arthrodesis: case series and literature review. J Spinal Disord Tech.17 (4):251-64.

Argenson C, Lassale B. (1996). Les fractures récentes du rachis thoracique et lombaire avec et sans troubles neurologiques. Rev chir orthop. 82 (1): 63117. 
Been HD, Bouma GJ. (1999). Comparison of two types of surgery for thoracolumbar burst fractures: combined anterior and posterior stabilisation vs. posterior instrumentation only. Acta Neurochir (Wien).141:349-57.

Chen Z, YI H, LI M, et al. (2011). Associations between body mass and the outcome of surgery for scoliosis in Chinese adults. PLoS One. 7: e21601.

Christodoulou A, Ploumis A, Terzidis I, Pournaras I. (2005). Vertebral body reconstruction with injectable hydroxyapatite cement for the management of unstable thoracolumbar burst fractures: a preliminary report. Acta Orthop Belg. 71:597-603.

Defino Hla, Rodriguez-Fuentes AE. (1998). Treatment of the thoracolumbar spine by anteroposterior fixation using the Harms method. Eur Spine J. 7: 187-94.

Denis F. (1983). The three column spine and its significance in the classification of a cute thoracolumbar spinal injuries. Spine. 8: 817-31.

Esses SI, Botsford DJ, Kostuik JP. (1990). Evaluation of surgical treatment for burst fractures. Spine. 15:667- 73.

Haas N, Blauth M, Tscherne H. (1991). Anterior plating in thoracolumbar spine injuries - indication, technique, and results. Spine. 16: S101-11.

Hangai M, Kaneoka K, Kuno S, Hinotsu S, Sakane M, et al. (2008). Factors associated with lumbar intervertebral disc degeneration in the elderly. Spine J. 8:732-40.

Hitchon P, Torner J, Eichholz K, Beeler S. (2006). Comparison of anterolateral and posterior approaches in the management of thoracolumbar burst fractures. J Neurosurg Spine. 5:117-25.

Lazannec JY, Neves N, Rousseau MA, et al. (2006). Wedge osteotomy for treating post-traumatic kyphosis at thoracolumbar and lumbar levels. $J$ Spinal Disord Tech. 19:487-94.

Lindsey RW, Dick W, Burns S, Zach G. (1991). The fixateur interne in the reduction and stabilisation of the thoracolumbar spine fractures in patients without neurological deficit. Spine. 16: S140-5.

Magerl F, Aebi M, Gertzabein SD, Harms J, Nazarian S. (1994). A comprehensive classification of thoracic and lumbar injuries. Eur Spine J. 3:184-201.

Muller U, Berlemann U, Sledge J, Schwarzenbach O. (1999). Treatment of thoracolumbar burst fractures without neurologic deficit by indirect reduction and posterior instrumentation: bisegmental stabilization with monosegmental fusion. Eur Spine J. 8:284-9.

Upasani VV, Calotum C, Petcharaporn M, et al. (2008). Does obesity affect surgical outcomes in adolescent idiopathic scoliosis. Spine (Phila Pa 1976). 33:e295-300. 
Wang XY, Dai LY, Xu HZ, Chi YL. (2008). Kyphosis recurrence after posterior short-segment fixation in thoracolumbar burst fractures. $J$ Neurosurg Spine. 8(3):246-54.

Wilcox RK, Boerger TO, Allen DJ, Barton DC, Limb D, Dickson RM, Hall RM. (2003). A dynamic study of thoracolumbar burst fractures. J Bone Joint Surg Am. 85: 2184-9.

Willen J, Anderson J, Toomoka K, Ssinger K. (1990). The natural history of burst fractures at the thoracolumbar junction. J Spinal Disord. 3(1): 39-46.

\section{Tableaux}

Tableau I : variation des paramètres en post-opératoire et au recul en fonction du montage chez les 30 patients. The modification of postoperative and follow up parameters according to the mounting length in 30 patients.

Montage long (16 patients)

Montage court (14 patients)

\begin{tabular}{ccccccccc} 
& $\begin{array}{c}\mathrm{CV} \\
\left({ }^{\circ}\right)\end{array}$ & $\mathrm{Cobb}\left(^{\circ}\right)$ & $\begin{array}{c}\mathrm{DIP} \\
(\mathrm{mm})\end{array}$ & $\mathrm{ART}\left({ }^{\circ}\right)$ & $\mathrm{CV}\left(^{\circ}\right)$ & $\mathrm{Cobb}\left(^{\circ}\right)$ & $\mathrm{DIP}(\mathrm{mm})$ & $\mathrm{ART}\left({ }^{\circ}\right)$ \\
\hline Post-op & 5,6 & 6,1 & 67,9 & 7,2 & 6 & 6,7 & 68,1 & 7,8 \\
Recul & 8,1 & 11,3 & 65,5 & 12,4 & 10 & 13,7 & 65,5 & 14,8 \\
Perte & $\mathbf{2 , 5}$ & $\mathbf{5 , 2}$ & $\mathbf{2 , 4}$ & $\mathbf{5 , 2}$ & $\mathbf{4}$ & $\mathbf{7}$ & $\mathbf{2 , 6}$ & $\mathbf{7}$ \\
\hline
\end{tabular}

Tableau II : variation des paramètres en post-opératoire et au recul en fonction de l'indice de masse corporelle (IMC). The modification of postoperative and follow up parameters according the body mass index (BMI).

\begin{tabular}{|c|c|c|c|c|c|c|c|c|}
\hline & \multicolumn{4}{|c|}{ IMC inférieur à 25 (19 patients) } & \multicolumn{4}{|c|}{ IMC supérieur à 25 (11 patients) } \\
\hline & $\mathrm{CV}\left({ }^{\circ}\right)$ & $\begin{array}{c}\text { Cobb } \\
\left({ }^{\circ}\right)\end{array}$ & DIP & $\begin{array}{l}\text { ART } \\
\left({ }^{\circ}\right)\end{array}$ & $\mathrm{CV}\left({ }^{\circ}\right)$ & $\begin{array}{c}\text { Cobb } \\
\left({ }^{\circ}\right)\end{array}$ & $\mathrm{DIP}(\mathrm{mm})$ & $\operatorname{ART}\left({ }^{\circ}\right)$ \\
\hline Post-op & 5,2 & 5,7 & 68,1 & 7,2 & 6,4 & 7,1 & 67,9 & 7,8 \\
\hline $\begin{array}{l}1 \text { an ou } \\
\text { plus }\end{array}$ & 8,2 & 11,6 & 66,2 & 13,1 & 9,8 & 13,4 & 64,8 & 14,1 \\
\hline $\begin{array}{c}\text { Perte } \\
\text { moyenne }\end{array}$ & 3 & 5,9 & 1,9 & 5,9 & 3,4 & 6,3 & 3,1 & 6,3 \\
\hline
\end{tabular}




\section{Figures}

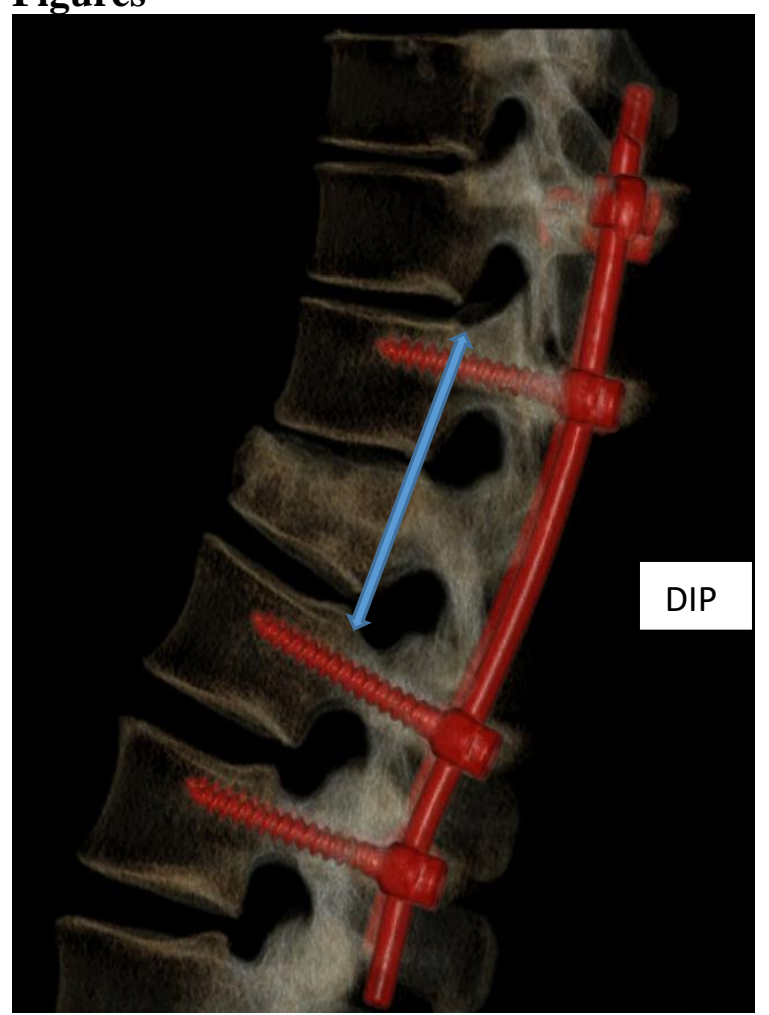

\section{Haut}

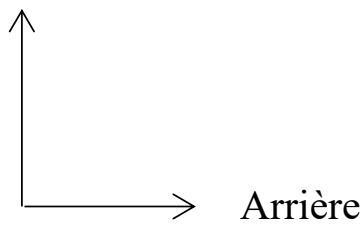

Figure 1 : mesure de la distance inter-pédiculaire (DIP). Method of measuring the interpedicular distance $(D I P)$. 